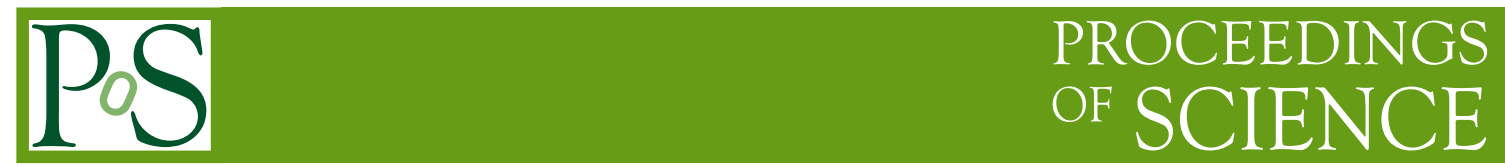

\title{
Numerical results for normal modes in lattice QCD
}

\author{
Wolfgang Söldner ${ }^{* a b}$ and Tilo Wettig ${ }^{a}$ \\ ${ }^{a}$ Institute for Theoretical Physics, University of Regensburg, 93040 Regensburg, Germany \\ ${ }^{b}$ Department of Physics, University of Bielefeld, 33615 Bielefeld, Germany \\ E-mail: soeldnerephysik.uni-bielefeld.de, \\ tilo.wettigephysik.uni-regensburg.de
}

\begin{abstract}
The normal modes of the Dirac eigenvalue spectrum are investigated in lattice QCD. We compare numerical results of lattice simulations to analytical predictions of chiral random matrix theory and obtain excellent agreement above a certain scale that is related to the boundary of the $\varepsilon$-regime of QCD.
\end{abstract}

XXIIIrd International Symposium on Lattice Field Theory

25-30 July 2005

Trinity College, Dublin, Ireland

${ }^{*}$ Speaker. 


\section{Introduction}

Random matrix theory (RMT) is a useful tool to analyze a wide variety of complex systems, see, e.g., the reviews [1, 国]. It can be used as a model to explain certain qualitative aspects of physical systems, but it also provides model-independent analytical results for universal properties of eigenvalue spectra. Here, we focus on the latter application of RMT. In a series of papers [3, 4, 可, Jackson and coworkers investigated the normal modes of the eigenvalue spectrum of various random matrix ensembles. These normal modes describe correlated fluctuations of the eigenvalues about their most probable values. They offer an independent way of understanding spectral correlations and serve as a complementary tool in the analysis of empirical eigenvalue spectra. In Ref. [7] it was suggested to subject the Dirac operator of lattice QCD to a normal-mode analysis. Such an analysis is the main subject of the present paper. We shall see that our results agree with the expectations of Ref. []]: most of the normal modes are described by RMT, but there are deviations from the random matrix result for normal modes with long wavelengths. The scale at which these deviations start to occur are related to the boundary of the $\varepsilon$-regime of QCD [6]. In condensed matter physics, a related scale is known as the Thouless energy [7].

\section{Normal modes in chiral random matrix theory}

Chiral RMT is obtained by retaining the chiral structure of the QCD Dirac operator $\mathbb{D}$ and replacing its matrix elements by random numbers [8],

$$
\mathbb{I D} \rightarrow\left(\begin{array}{cc}
0 & i W \\
i W^{\dagger} & 0
\end{array}\right)
$$

We restrict ourselves to the physical case of gauge group SU(3) and fermions in the fundamental representation in which $W$ is a complex matrix of dimension $N \times(N+v)$ [9]. The matrix in Eq. (2.1) has $|v|$ eigenvalues equal to zero, thus $v$ can be interpreted as the topological charge. Without loss of generality, we take $v \geq 0$.

The nonzero eigenvalues of $i D$ come in pairs $\pm \lambda_{k}(k=1, \ldots, N)$. We are interested in the joint probability distribution $P_{N}\left(\lambda_{1}, \ldots, \lambda_{N}\right)$ of the eigenvalues $\lambda_{k}$. For the probability distribution of the elements of $W$ we choose a Gaussian, $\exp \left(-N \Sigma^{2} \operatorname{tr} W W^{\dagger}\right)$, and an additional factor of $\prod_{f=1}^{N_{f}} \operatorname{det}\left(\mathbb{D}+m_{f}\right)$ which corresponds to the fermion determinant due to $N_{f}$ flavors with masses $m_{f}$. In the following, we only consider massless flavors. The parameter $\Sigma$ denotes the absolute value of the chiral condensate. After a singular value decomposition of $W$, the joint probability distribution becomes

$$
P_{N}\left(\lambda_{1}, \ldots, \lambda_{N}\right)=\mathscr{N} \Delta^{2}\left(\lambda^{2}\right) \prod_{k=1}^{N} \lambda_{k}^{2\left(N_{f}+v\right)+1} e^{-N \Sigma^{2} \lambda_{k}^{2}},
$$

where $\mathscr{N}$ is a normalization constant and $\Delta$ is the Vandermonde determinant, $\Delta(x)=\prod_{i<j}\left(x_{i}-x_{j}\right)$. We see from Eq. (2.2) that $N_{f}$ and $v$ only occur in the combination $N_{f}+v$, i.e., a massless flavor can be traded for one unit of topological charge. We can therefore set $N_{f}=0$ and only retain $v$. For further reference, we note that the global spectral density of the random matrix model (2.1) is given by the Wigner semicircle, $\rho(\lambda)=(2 N \Sigma / \pi)\left(1-(\Sigma \lambda / 2)^{2}\right)^{-1 / 2}$. 
We assume that the $\lambda_{k}$ are ordered with $0 \leq \lambda_{1} \leq \ldots \leq \lambda_{N}$. The most probable locations of the eigenvalues are given by the maxima of $P_{N}$. These are obtained when the eigenvalues are located at the zeros of Laguerre polynomials, see Ref. [5] for details. Since $P_{N}$ is symmetric in its arguments, it suffices to consider a single maximum, denoted by $P_{N}^{0}$. Near this maximum, the logarithm of $P_{N}$ can be approximated by

$$
\ln P_{N} \approx \ln P_{N}^{0}+\frac{1}{2} \sum_{i j} \delta \lambda_{i} C_{i j} \delta \lambda_{j}
$$

with a real symmetric matrix $C$ given in Ref. [5]. The eigenvalues of this matrix are

$$
\omega_{k}=-4 k N \Sigma^{2}
$$

with $k=1, \ldots, N$. Note that the $\omega_{k}$ are independent of $v$. The eigenvectors $\phi^{(k)}$ of $C$, whose explicit form can be found in Ref. [5], are the normal modes of the random matrix spectrum. They are statistically independent and describe correlated fluctuations of the eigenvalues about their most probable values. The eigenvalues $\omega_{k}$ measure the magnitude of these fluctuations. The main focus of the present paper is a comparison of the normal mode spectrum of the lattice Dirac operator with the analytical result (2.4), and the interpretation of deviations from this result.

\section{Unfolding and normal modes in empirical spectra}

In Secs. 4 and 5 we will present ensembles of numerical eigenvalue spectra obtained in RMT and in lattice QCD, respectively. In order to compare these data to universal analytical predictions of RMT, the dependence of the global level density $\rho$ on system-specific details must be eliminated. This is done by an unfolding procedure in which the energies $E$ are rescaled according to

$$
E \rightarrow \xi(E)=\int_{-\infty}^{E} d E^{\prime} \rho\left(E^{\prime}\right)
$$

By construction, the mean level spacing or, equivalently, the mean level density, of the unfolded energies $\xi$ is equal to unity. The quantity on the right-hand side of Eq. (3.1) is called the staircase function. There are two different methods of determining $\rho(E)$, known as spectral averaging and ensemble averaging, respectively. They can potentially lead to different results because they probe different energy scales of the spectra. For the present application, the natural choice is ensemble averaging, see Refs. [4, 10] for details.

After unfolding, a single spectrum consists of $N$ eigenvalues $x_{1}, \ldots, x_{N}$ with $\left\langle x_{i}\right\rangle=i$, where $\langle\cdots\rangle$ denotes an ensemble average. To investigate the normal mode spectrum, i.e., the fluctuations of the eigenvalues about their average positions, we construct the real and symmetric correlation matrix [3]

$$
D_{i j}=\left\langle\left(x_{i}-\left\langle x_{i}\right\rangle\right)\left(x_{j}-\left\langle x_{j}\right\rangle\right)\right\rangle=\left\langle x_{i} x_{j}\right\rangle-\left\langle x_{i}\right\rangle\left\langle x_{j}\right\rangle
$$

whose eigenvalues will be denoted by $\alpha_{k}$.

Within RMT, the matrices $C$ and $D$ are related. If the small-amplitude approximation in Eq. (2.3) holds, the eigenvectors of $C$ and $D$ are identical, and the eigenvalues of $D$ and $C$ are related by $\alpha_{k}^{\mathrm{RMT}}=-1 / \tilde{\omega}_{k}$, where $\tilde{\omega}_{k}$ is the dispersion relation of the normal modes on the unfol-

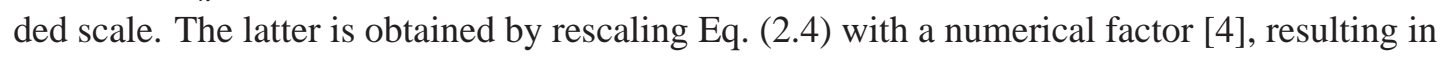

$$
\alpha_{k}^{\mathrm{RMT}}=-\frac{1}{\tilde{\omega}_{k}}=\frac{N}{4 \sqrt{2} \pi k} .
$$



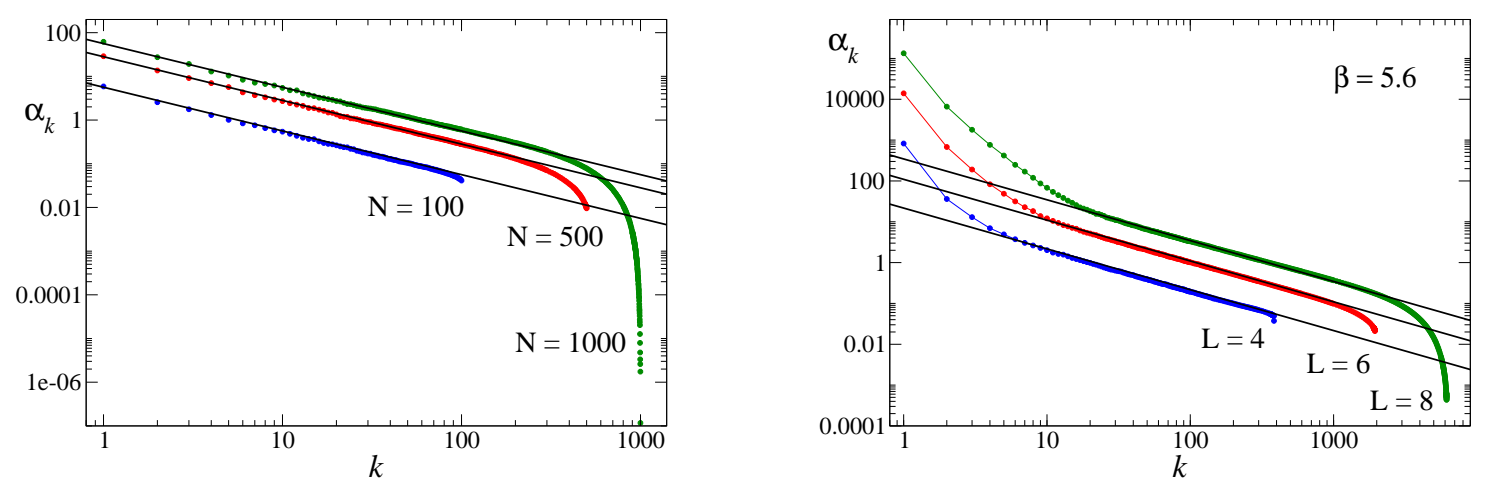

Figure 1: The data points in the plot on the left-hand side represent the eigenvalues of the correlation matrix (3.2) obtained from a numerical simulation of the random matrix model (2.1) for three values of the matrix size $N$. In the plot on the right-hand side, the data points represent the eigenvalues of the correlation matrix (3.2) obtained from the staggered lattice Dirac operator 5.1) for three values of the lattice size $V=L^{4}$. In both plots, the straight lines are the analytical prediction (3.3).

\section{Chiral GUE}

We first present numerical results for the chiral Gaussian unitary ensemble (GUE) of RMT, i.e., the random matrix model of Eq. (2.1). We set $v=0$ and $\Sigma=1$ and generated three ensembles of 1000 configurations each for $N=100,500$, and 1000, respectively. The eigenvalue spectra were unfolded using the numerically determined staircase function for each ensemble, as described in Sec. 3. We then constructed the correlation matrix of Eq. (3.2) from the unfolded eigenvalues. The eigenvalues of this matrix are plotted on the left-hand side of Fig. 1, along with the analytical prediction of Eq. (3.3). We observe excellent agreement for small $k$ and deviations for large $k$. These deviations are due to limited statistics. Increasing the number of configurations improves the agreement between data and analytical prediction, and infinite statistics would result in perfect agreement.

\section{Lattice QCD}

We have computed eigenvalue spectra of the unimproved staggered Dirac operator,

$$
\mathbb{D}=\frac{1}{2 a} \sum_{\mu=1}^{4} \alpha_{\mu}(x)\left[\delta_{y, x+\hat{\mu}} U_{\mu}(x)-\delta_{y, x-\hat{\mu}} U_{\mu}^{\dagger}(y)\right]
$$

where $a$ is the lattice spacing and $U_{\mu}(x)$ and $\alpha_{\mu}(x)$ denote the link variables and the staggered phases, respectively. The gauge action is the standard Wilson plaquette action. In our simulation we have used lattice volumes $V=4^{4}, 6^{4}$ and $8^{4}$ at $\beta=5.6$ with 7000 configurations each. The number of distinct positive eigenvalues of $i \mathbb{D}$ on a lattice with $V$ sites is $N=3 \mathrm{~V} / 2$.

The unimproved staggered Dirac operator does not have exact zero modes at finite lattice spacing even if the gauge field has nontrivial topological charge. At the value of $\beta=5.6$, the $\mathscr{O}\left(a^{2}\right)$ shift of the would-be zero modes is so large that they are completely mixed up with the nonzero modes. As a result, the eigenvalue spectrum is effectively in the $v=0$ sector. This is 

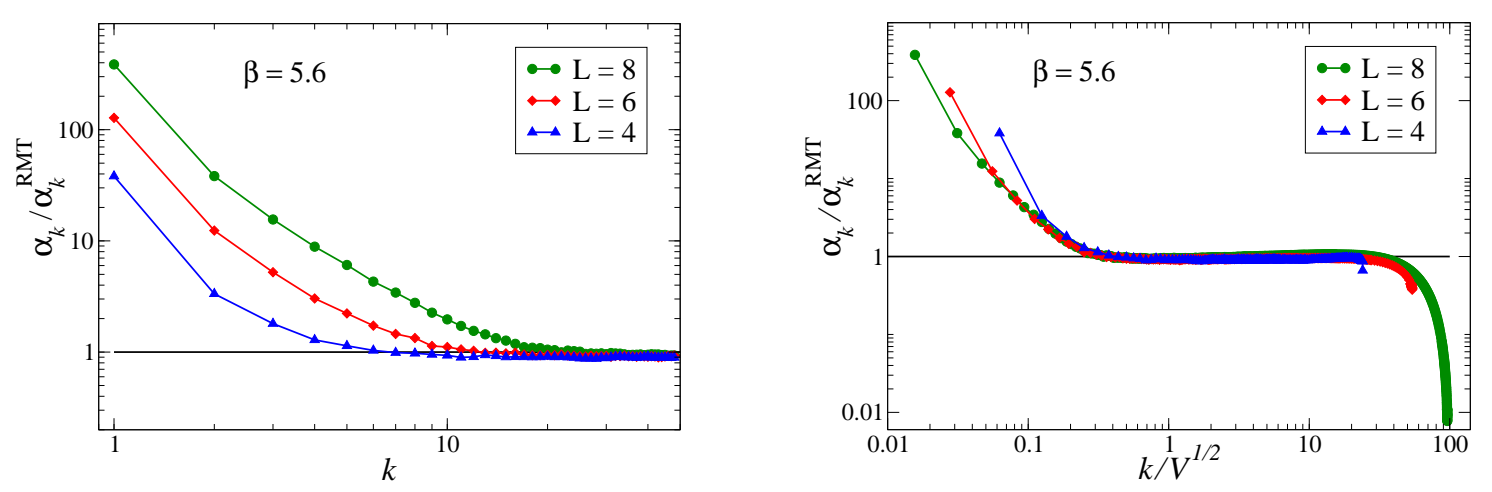

Figure 2: On the left-hand side, we show the ratio of the lattice data for $\alpha_{k}$ and the corresponding RMT prediction $\alpha_{k}^{\mathrm{RMT}}$ plotted vs $k$. On the right-hand side, we show the same ratio but now plotted vs $k / \sqrt{V}$.

a well known problem with a long history [11, 12], and recent studies have shown that it can be alleviated by various improvement and smearing schemes [13, 14, 15, 16]. In our present work, we are not concerned with this issue since the RMT prediction of Eq. (3.3) does not depend on $v$.

The numerically obtained eigenvalue ensembles were unfolded and the correlation matrix $D$ was constructed and diagonalized as in Sec. 7 . In the plot on the right-hand side of Fig. 1, we show the eigenvalues of $D$ as a function of $k$ along with the analytical RMT prediction. As in the case of the chiral GUE, we again observe characteristic deviations for large $k$ that are due to limited statistics. What is more interesting, however, are the deviations between lattice data and RMT for small $k$. These deviations, which indicate a genuine breakdown of RMT, will now be investigated in more detail.

\section{Thouless energy}

In Fig. 目 we show the same data as in the plot on the right-hand side of Fig. 1, but we now plot the ratio of the lattice data for $\alpha_{k}$ and the corresponding RMT prediction (with $N=3 \mathrm{~V} / 2$ ). In the plot on the left-hand side we magnified the region of small $k$. As the wave number $k$ decreases, there is a "critical" value $k_{c}$ at which the lattice data start to deviate from RMT. We see that this critical value increases with $V$. In the plot on the right-hand side of Fig. 目, we rescaled $k \rightarrow k / \sqrt{V}$. We see that the three rescaled curves nicely fall on top of each other and start to deviate from RMT at the same value of $k / \sqrt{V}$ (the deviations for large $k$ are due to limited statistics as explained above). This means that $k_{c}$ scales with $\sqrt{V}$ as expected [4. 5]. It reflects the fact that the spectral correlations of the Dirac operator are described by RMT only in the $\varepsilon$-regime of QCD in which the zero-momentum modes of the Goldstone fields dominate the partition function [6]. The boundary of this regime, which in analogy to a similar situation in condensed matter physics is called the Thouless energy $E_{c}$ [7], scales as $E_{c} / \Delta \sim \sqrt{V}$, where $\Delta$ is the mean level spacing of the Dirac operator at zero virtuality [17]. Clearly, $k_{c}$ and $E_{c}$ are intimately related, but we currently do not know the precise relationship between these two quantities. Once such a relationship is established, the deviations of the lattice data for $\alpha_{k}$ from the RMT prediction will provide another method to estimate the Thouless energy in lattice QCD. 


\section{Conclusions}

We have shown that the normal mode spectrum of the lattice Dirac operator is described by the analytical prediction of RMT for wave numbers $k$ larger than a critical wave number $k_{c}$. This critical wave number scales with $\sqrt{V}$ and is related to the boundary of the $\varepsilon$-regime of QCD. In future work, it would be desirable to find a precise relationship between $k_{c}$ and the Thouless energy $E_{c}$. To this end, we plan to investigate the dependence of $k_{c}$ on the lattice coupling $\beta$.

\section{Acknowledgments}

This work was supported in part by DFG and BMBF. WS acknowledges the hospitality of Brookhaven National Laboratory, where part of this work was performed.

\section{References}

[1] T. Guhr, A. Müller-Groeling, and H.A. Weidenmüller, Phys. Rep. 299 (1998) 189 [cond-mat/9707301]

[2] J.J.M. Verbaarschot and T. Wettig, Annu. Rev. Nucl. Part. Sci. 50 (2000) 343 hep-ph/0003017]

[3] A. Andersen, A.D. Jackson, and H.J. Pedersen, Nucl. Phys. A 650 (1999) 213 [nucl-th/9812037]

[4] A.D. Jackson et al., Nucl. Phys. A 687 (2001) 405 [cond-mat/0009332]

[5] A.D. Jackson, C.B. Lang, M. Oswald, and K. Splittorff, Nucl. Phys. B 616 (2001) 233 [hep-th/0108141]

[6] J. Gasser and H. Leutwyler, Phys. Lett. B 188 (1987) 477

[7] D.J. Thouless, Phys. Rep. 13 (1974) 93, J. Phys. C 8 (1975) 1803, Phys. Rev. Lett. 39 (1977) 1167

[8] E.V. Shuryak and J.J.M. Verbaarschot, Nucl. Phys. A 560 (1993) 306 [hep-th/9212088

[9] J.J.M. Verbaarschot, Phys. Rev. Lett. 72 (1994) 2531 [hep-th/9401059]

[10] T. Guhr, J.-Z. Ma, S. Meyer, and T. Wilke, Phys. Rev. D 59 (1999) 054501 hep-lat/9806003]

[11] J. Smit and J.C. Vink, Nucl. Phys. B 286 (1987) 485

[12] M.E. Berbenni-Bitsch et al., Phys. Rev. Lett. 80 (1998) 1146 [hep-lat/9704018]

[13] E. Follana, A. Hart, and C.T.H. Davies, Phys. Rev. Lett. 93 (2004) 241601 hep-lat/0406010]

[14] S. Dürr, C. Hoelbling, and U. Wenger, Phys. Rev. D 70 (2004) 094502 hep-lat/0406027]

[15] K.Y. Wong and R.M. Woloshyn, Phys. Rev. D 71 (2005) 094508 hep-lat/0412001

[16] E. Follana, A. Hart, C.T.H. Davies, and Q. Mason, hep-lat/0507011

[17] J.C. Osborn and J.J.M. Verbaarschot, Nucl. Phys. B 525 (1998) 738 [hep-ph/9803419, Phys. Rev. Lett. 81 (1998) 268 [hep-ph/9807490]; R.A. Janik, M.A. Nowak, G. Papp, and I. Zahed, Phys. Rev. Lett. 81 (1998) 264 [hep-ph/9803289], Phys. Lett. B 442 (1998) 300 [hep-ph/9807550]; M.E. Berbenni-Bitsch et al., Phys. Lett. B 438 (1998) 14 [hep-ph/9804439] 